\title{
Cerebrovascular reactivity is not associated with therapeutic intensity in adult traumatic brain injury: a CENTER-TBI analysis
}

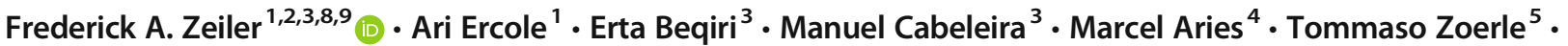 \\ Marco Carbonara ${ }^{5}$ - Nino Stocchetti ${ }^{5,6}$ - Peter Smielewski ${ }^{3}$. Marek Czosnyka ${ }^{3,7}$. David K. Menon ${ }^{1}$ - the CENTER-TBI \\ High Resolution ICU (HR ICU) Sub-Study Participants and Investigators
}

Received: 3 May 2019 / Accepted: 11 June 2019 / Published online: 25 June 2019

(C) The Author(s) 2019

\begin{abstract}
Background Impaired cerebrovascular reactivity in adult traumatic brain injury (TBI) is known to be associated with poor outcome. However, there has yet to be an analysis of the association between the comprehensively assessed intracranial hypertension therapeutic intensity level (TIL) and cerebrovascular reactivity.

Methods Using the Collaborative European Neuro Trauma Effectiveness Research in TBI (CENTER-TBI) high-resolution intensive care unit (ICU) cohort, we derived pressure reactivity index (PRx) as the moving correlation coefficient between slow-wave in ICP and mean arterial pressure, updated every minute. Mean daily PRx, and daily $\%$ time above PRx of 0 were calculated for the first 7 days of injury and ICU stay. This data was linked with the daily TIL-Intermediate scores, including total and individual treatment sub-scores. Daily mean PRx variable values were compared for each TIL treatment score via mean, standard deviation, and the Mann $U$ test (Bonferroni correction for multiple comparisons). General fixed effects and mixed effects models for total TIL versus PRx were created to display the relation between TIL and cerebrovascular reactivity. Results A total of 249 patients with 1230 ICU days of high frequency physiology matched with daily TIL, were assessed. Total TIL was unrelated to daily PRx. Most TIL sub-scores failed to display a significant relationship with the PRx variables. Mild hyperventilation $(p<0.0001)$, mild hypothermia $(p=0.0001)$, high levels of sedation for ICP control $(p=0.0001)$, and use vasopressors for CPP management $(p<0.0001)$ were found to be associated with only a modest decrease in mean daily PRx or $\%$ time with PRx above 0 .

Conclusions Cerebrovascular reactivity remains relatively independent of intracranial hypertension therapeutic intensity, suggesting inadequacy of current TBI therapies in modulating impaired autoregulation. These findings support the need for
\end{abstract}

This article is part of the Topical Collection on Brain trauma

Electronic supplementary material The online version of this article (https://doi.org/10.1007/s00701-019-03980-8) contains supplementary material, which is available to authorized users.

Frederick A. Zeiler

umzeiler@myumanitoba.ca

Ari Ercole

ae105@cam.ac.uk

Erta Beqiri

erta.beqiri@gmail.com

Manuel Cabeleira

mc916@cam.ac.uk

Marcel Aries

marcel.aries@mumc.nl

Tommaso Zoerle

tommaso.zoerle@policlinico.mi.it

\author{
Marco Carbonara \\ marco.carbonara@gmail.com \\ Nino Stocchetti \\ nino.stocchetti@policlinico.mi.it \\ Peter Smielewski \\ ps10011@cam.ac.uk \\ Marek Czosnyka \\ mc141@medschl.cam.ac.uk \\ David K. Menon \\ dkm13@cam.ac.uk
}

Extended author information available on the last page of the article 
investigation into the molecular mechanisms involved, or individualized physiologic targets (ICP, CPP, or Co2) in order to treat dysautoregulation actively.

Keywords Cerebrovascular reactivity $\cdot \mathrm{PRx} \cdot \mathrm{TBI} \cdot$ Therapeutic intensity $\cdot \mathrm{TIL}$

\section{Introduction}

Impaired cerebrovascular reactivity after traumatic brain injury (TBI) has emerged as a meaningful independent factor associated with mortality and poor functional outcome at 6 and 12 months post-injury [5, 20, 27, 28]. Current methodology for continuous measurement of cerebrovascular reactivity employed in the intensive care unit (ICU) is based on calculation of a moving correlation between slow-wave vasogenic fluctuations pulsatile cerebral blood volume (using the surrogate of intracranial pressure (ICP)), and a measure of driving pressure (such as mean arterial pressure (MAP)) [5, 26].

The pressure reactivity index (PRx), derived from ICP and MAP, is the most commonly quoted index of cerebrovascular reactivity in the TBI literature [26]. This particular index has experimental support as a potential measure of the lower limit of autoregulation [3]. Further, in adult TBI, PRx has defined critical thresholds associated with poor outcomes [20, 27].

However, despite the strong relationship between autoregulation and outcome, currently available ICU therapies do not allow us to modulate cerebrovascular reactivity as a pathway to improving outcome. A recent retrospective analysis assessed the last 25 years of experience with therapies guided by cerebral physiology monitoring in adult TBI. This analysis suggested that, despite changes in Brain Trauma Foundation (BTF) ICP and cerebral perfusion pressure (CPP) targets, little to no impact on PRx was seen [7]. This limited change in monitored PRx occurred in parallel to a stable mortality level in the same cohort over the 25 -year period. This corroborates similar findings from a smaller study of 48 TBI patients, evaluating factors impacting the ability to calculate CPP optimum from PRx [24]. While these findings suggest that PRx is independent of current TBI therapies, they require robust corroboration in an analysis that accounts for the intensity of therapy. If validated, they suggest the need for further work on the molecular mechanisms or complex individual physiology involved in impaired cerebrovascular reactivity, so as to aid in the development of targeted therapies aimed at enhancing autoregulatory reserve, with the aim of increasing resilience to physiological insults and improving outcomes.

The goal of this project was to assess the association between daily treatment intensity, as measured through the therapeutic intensity level (TIL) intermediate scoring system [12, 31], and daily measures of cerebrovascular reactivity, using the Collaborative European Neuro Trauma Effectiveness Research in TBI (CENTER-TBI) study high-resolution ICU sub-study cohort [13].

\section{Methods}

\section{Patient population}

All patients from the multi-center CENTER-TBI highresolution ICU cohort were included for this study. These patients were prospectively recruited between January 2015 and December 2017, from 21 centers in the European Union (EU). All patients were admitted to ICU for their TBI during the course of the study, with high-frequency digital signals recorded from their ICU monitors during the course of their ICU stay. All patients suffered predominantly from moderate to severe TBI (moderate $=$ Glasgow Coma Score $(\mathrm{GCS}) 9$ to 12 , and severe $=$ GCS of 8 or less). A minority of patients suffered from non-severe TBI, with subsequent early deterioration leading to ICU admission for care and monitoring. All patients in this cohort had invasive ICP monitoring conducted in accordance with the BTF guidelines [4].

Ethics Data used in these analyses were collected as part of the CENTER-TBI study which had individual national or local regulatory approval; the UK Ethics approval is provided as an exemplar (IRAS No. 150943; REC 14/SC/1370). The CENTER-TBI study (EC grant 602150) has been conducted in accordance with all relevant laws of the EU if directly applicable or of direct effect and all relevant laws of the country where the recruiting sites were located, including but not limited to, the relevant privacy and data protection laws and regulations (the "Privacy Law"), the relevant laws and regulations on the use of human materials, and all relevant guidance relating to clinical studies from time to time in force including, but not limited to, the ICH Harmonised Tripartite Guideline for Good Clinical Practice (CPMP/ICH/135/95) ("ICH GCP") and the World Medical Association Declaration of Helsinki entitled "Ethical Principles for Medical Research Involving Human Subjects". Informed consent by the patients and/or the legal representative/next of kin was obtained, accordingly to the local legislations, for all patients recruited in the core dataset of CENTER-TBI and documented in the e-CRF.

\section{Data collection}

As part of recruitment to the multi-center high-resolution ICU cohort of CENTER-TBI [13], demographics and clinical data were prospectively collected and had high-frequency digital 
signals from ICU monitoring recorded throughout their ICU stay, with the goal of initiating recording within $24 \mathrm{~h}$ of injury. All digital ICU signals were further processed (see signal acquisition/signal processing). For the purpose of this study, the following admission demographic variables were collected: age, sex, and admission Glasgow Coma Scale (GCS, total and motor). Finally, daily total TIL and all daily TIL subscores were also collected for each patient for the first 7 days after injury. Of note, not all patients had a full 7 days of TIL scoring given different clinical trajectories. Appendix A provides a breakdown of the TIL intermediate scoring system and its sub-score components. CENTER-TBI data version 1.0 was accessed for the purpose of this study, via Opal database software [6].

\section{Signal acquisition}

Arterial blood pressure (ABP) was obtained through either radial or femoral arterial lines connected to pressure transducers. ICP was acquired via an intra-parenchymal strain gauge probe (Codman ICP MicroSensor; Codman \& Shurtleff Inc., Raynham, MA), parenchymal fiber optic pressure sensor (Camino ICP Monitor, Integra Life Sciences, Plainsboro, NJ, USA; https://www.integralife.com/) or external ventricular drain. All signals were recorded using digital data transfer or digitized via an A/D converter (DT9801; Data Translation, Marlboro, MA), where appropriate, sampled at frequency of $100 \mathrm{~Hz}(\mathrm{~Hz})$ or higher, using the ICM+ software (Cambridge Enterprise Ltd., Cambridge, UK; http://icmplus.neurosurg. cam.ac.uk) or Moberg CNS Monitor (Moberg Research Inc., Ambler, PA, USA) or a combination of both. Signal artifacts were removed using automated methods prior to further processing or analysis.

\section{Signal processing}

Post-acquisition processing of the above signals was conducted using ICM+. CPP was determined as CPP = MAP - ICP. Ten-second moving averages (updated every $10 \mathrm{~s}$ to avoid data overlap) were calculated for all recorded signals: ICP, ABP (which produced MAP), and CPP.

PRx was derived using the Pearson correlation between 30 consecutive $10 \mathrm{~s}$ mean values for ICP and MAP, updated every minute. Data were provided in minute-by-minute comma-separated variable sheets for the entire duration of recording for each patient.

\section{Data processing}

Post-ICM+ processing was undertaken using R ( $\mathrm{R}$ Core Team (2016). R: A language and environment for statistical computing. R Foundation for Statistical Computing, Vienna, Austria.
URL https://www.R-project.org/). Using the date and time stamp for each minute-by-minute data point, daily summaries were provided for days 1 through 7 after injury for each patient, producing daily mean ICP, daily \% time with ICP above $20 \mathrm{mmHg}$, daily \% time with ICP above $22 \mathrm{mmHg}$, daily mean PRx, and daily \% time spent above PRx of 0 . This PRx threshold was selected given previous literature confirming its relationship with global patient outcome [20], and belief that is represents the PRx value where vascular reactivity begins becoming impaired [3], representing phaseshift between slow-waves in ICP and MAP trending below $90^{\circ}$. These daily physiologic measures were then linked with the daily TIL measures for statistical analysis. These data are referred to as the "day-matched" data.

A second data sheet was also produced using time-shifted data. We were interested in assessing the impact of TIL score on the following-day cerebrovascular reactivity. As such, we time-shifted the TIL and physiology data in order to assess this relationship. We refer to this data as the "time-shifted" data.

\section{Statistics}

All statistical analysis was conducted using $\mathrm{R}$ and XLSTAT (Addinsoft, New York, NY; https:/www.xlstat.com/en/) addon package to Microsoft Excel (Microsoft Office 15, Version 16.0.7369.1323). Normality of continuous variables was assessed via the Shapiro-Wilks test.

Box plots were used to visualize the relationship between ICP and PRx variables across increasing TIL total and subscores. Using total TIL score, mean ICP, and PRx variable values were compared across increasing TIL, using the Jonckheere-Terpstra test, with 1000 permutations, with alpha set at 0.05 for this test. Similarly, mean PRx variable values were compared across each TIL sub-score, with alpha set at 0.0005 after the Bonferroni correction for multiple comparisons. Given that almost all TIL sub-scores failed to demonstrate statistical significance in association with PRx variables, only the statistically significant associations are reported in detail in the manuscript. Identical results were found for mean daily PRx, and mean daily \% time above PRx of 0 . Similarly, identical results were found for both the day-matched and time-shifted data sheets.

In order to highlight the association between cerebrovascular reactivity with therapeutic intensity, we derived both general linear fixed effects and linear mixed effects (LME) models for total TIL versus: mean daily PRx and daily \% time with PRx above 0. Patient examples were used to confirm no autocorrelation between daily physiologic measures, using autocorrelation function (ACF) and partial autocorrelation function (PACF) plots. Thus, no autocorrelative structure for the PRx variables was included in these models. Both the generalized and LME models were to be compared for 
superiority via the Akaike information criterion (AIC), the Bayesian information criterion (BIC), and analysis of variance (ANOVA). Alpha for ANOVA testing was set at 0.05. Given all LME and generalized fixed effects models failed to reach significance for the relationship between total TIL and cerebrovascular reactivity metrics, these models are not reported in detail within the manuscript, and only mentioned in reference to the global findings of this study.

Finally, in order to determine if there is a difference in response of PRx measures to TIL therapies during the more acute initial phase of ICU stay, we evaluated all of the above in the first 3 days of recording. Of note, none of the results were different using the first 3 days, versus the first 7 days of ICU stay. As such, we report only the first 7 days of ICU stay within this manuscript, making reference to the first 3 days analysis intermittently throughout.

\section{Results}

\section{Patient demographics}

There were 249 patients from the CENTER-TBI high-resolution ICU cohort, for whom high-frequency physiologic signals and daily TIL scores were available, and were included in this study. An account of general patient characteristics can be found in other publications on this high-resolution data cohort $[28,29]$. A total of 1230 daily observations of TIL and daily PRx variables were used for this analysis. The mean age was $46.8 \pm 18.9$ years, with 198 being male. Median admission GCS was 6 (IQR, 3 to 10), median admission GCS motor score was 4 (IQR, 1 to 5), and median duration of physiologic monitoring was $140.6 \mathrm{~h}$ (IQR, 94.4 to 213.6). All continuous variables were found to be non-parametrically distributed. Mean daily ICP and PRx variable values across the patient population remained relatively constant, with mean daily ICP well below BTF defined thresholds and mean daily $\%$ time with PRx above 0 remaining consistently above 40 to $50 \%$. There was only a significant drop in \% time with PRx above zero going from day 1 (i.e., first $24 \mathrm{~h}$ after injury) to day 2 post-injury ( $p=0.03$; Mann $U$ ), and going from day 2 to day 3 ( $p=0.004$; Mann $U$ ), with no difference between days 3 and 7. A similar relationship was seen for $\%$ time with ICP above $20 \mathrm{mmHg}$, \% time with ICP above $22 \mathrm{mmHg}$. Figure 1 displays boxplots for the mean daily ICP and mean daily $\%$ time with PRx above 0 .

\section{Daily total TIL and cerebrovascular reactivity}

Comparing total daily TIL to the daily measures of ICP and $\mathrm{PRx}$, the same relationships were seen for the day-matched and time-shifted data, using both the first 7 days and first 3 days of data. With increasing total daily TIL, there was an increase in daily mean ICP, $\%$ time above ICP of $20 \mathrm{mmHg}$ and $\%$ time above ICP of $22 \mathrm{mmHg}(p=0.001$ on Jonckheere Terpstra testing for increasing values in all); in keeping with escalating daily therapy for increasing daily ICP measures. In contrast, there was no relationship between increasing daily total TIL and daily measures of PRx (mean values or \% time above threshold; $p>0.05$ on Jonckheere-Terpstra test for increasing and decreasing values for all), in both the daymatched and time-shifted data sheets. This suggests potentially limited treatment effect of daily TIL measured therapies on daily cerebrovascular reactivity metrics. Figure 2 displays the boxplots of total daily TIL and both daily \% time with PRx above 0 and mean daily ICP, for both the day-matched and time-shifted data.

Generalized and LME models were created to assess the relationship between total TIL and mean daily PRx and daily $\%$ time with PRx above 0 . All models failed to display statistically significant relationships with total TIL, even with the introduction of random effects to both the intercept and gradient by patient $(p<0.05$ for all). As such these models will not be reported further here, but provide confirmatory evidence for the lack of association between TIL and cerebrovascular reactivity metrics.

\section{Daily TIL sub-scores and cerebrovascular reactivity}

Comparing daily TIL sub-scores to daily measures of PRx, identical relationships were seen for all PRx variables (i.e. ,mean PRx, \% time with PRx $>0$ ), in both the day-matched and time-shifted datasets, using both the first 7 days and first 3 days of data. Only 4 daily TIL sub-score measures displayed a statistically significant decrease in daily PRx measures using day-matched and time-shifted data on Mann $U$ testing: mild hyperventilation $\left(\mathrm{PaCO}_{2} 35\right.$ to $40 \mathrm{mmHg}$ ), mild hypothermia (core temp $>35{ }^{\circ} \mathrm{C}$ ), high sedation levels targeting ICP, and vasopressor therapy targeting CPP goals. Table 1 provides the Mann $U$ testing for $\%$ time with PRx above 0 for the 4 daily TIL sub-scores for both the day-matched and time-shifted data. Appendix B provides the boxplots for both the timematched and time-shifted data. Of note, the overall absolute reduction in daily \% time with PRx above 0 seen with these 4 interventions was less than $10 \%$, indicating a modest potential treatment.

\section{Discussion}

The analysis of the relationship between TIL and measures of cerebrovascular reactivity provided some interesting results which deserve highlighting. First, evaluating the $\%$ time spent with PRx above zero across the first 7 days of ICU stay, it can be seen that a large portion of any given day is spent with impaired cerebrovascular reactivity. There is statistically 
a $\quad \%$ Time with PRx $>0$ vs. Day From Injury

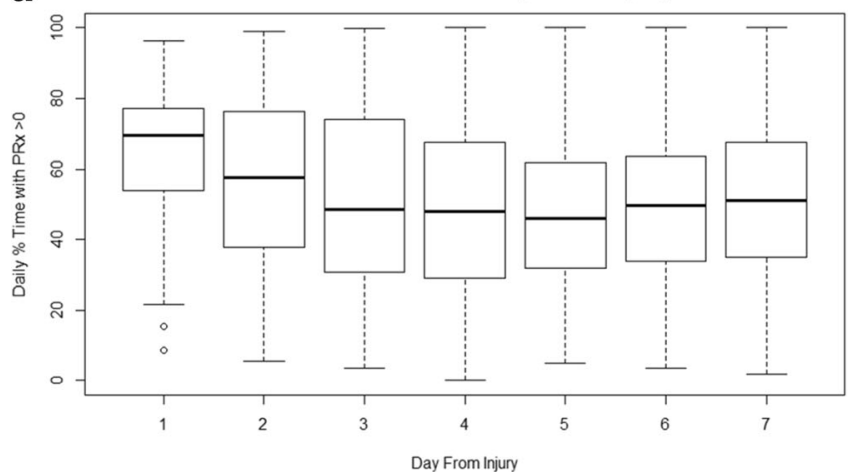

Fig. 1 Boxplots of mean daily ICP and \% time with $\mathrm{PRx}>0$ versus day. ICP, intra-cranial pressure; MAP, mean arterial pressure; $\mathrm{mmHg}$, millimeter of mercury; PRx, pressure reactivity index (moving correlation between ICP and MAP). Day from injury (i.e., day-matched

significant decrease in this value going from the first $24 \mathrm{~h}$ post injury to day 2 and day 3 in our cohort. However, from day 3 onwards, there is little change in the amount of time spent with PRx above zero, with no difference between days. As discussed below, this initial drop in time above PRx of zero is not accounted for in total TIL or specific TIL-based

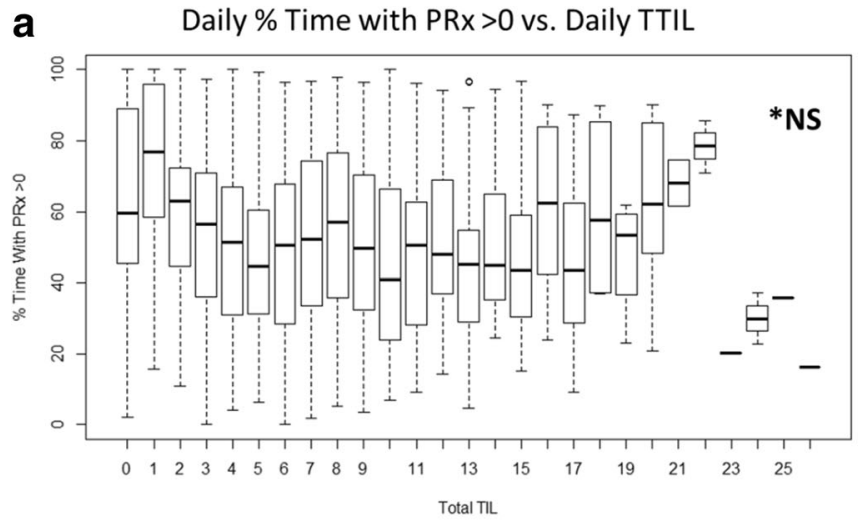

C

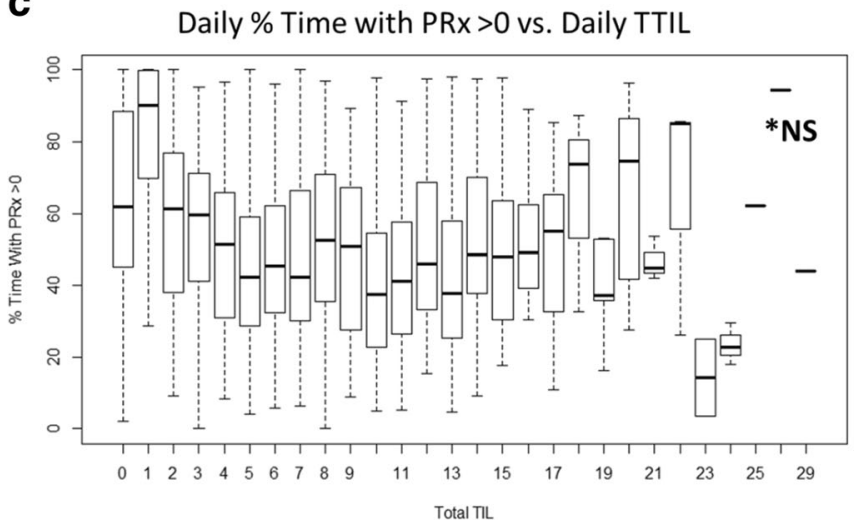

Fig. 2 Boxplots of mean daily ICP and mean daily \% time with PRx $>0$ versus daily total TIL. ICP, intra-cranial pressure; MAP, mean arterial pressure; $\mathrm{mmHg}$, millimeter of mercury; NS, non-significant; TIL, therapeutic intensity level; TTIL, total daily TIL. ${ }^{*} p$ values reported are for the Jonckheere-Terpstra test for increasing mean values. Relationship

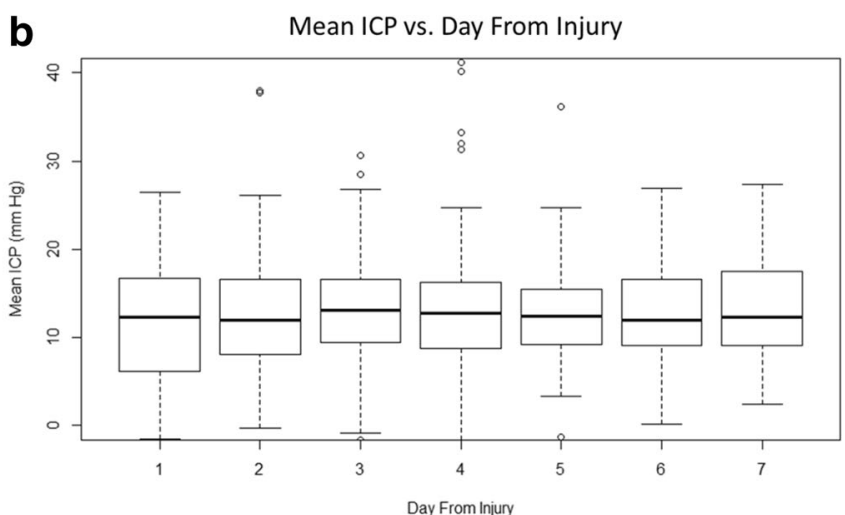

data), day $1=$ day of injury. Plot of daily $\%$ time with $P R x>0$ versus day from injury to day-matched data $\mathbf{a}$; Plot of mean daily ICP versus day from injury to day-matched data $\mathbf{b}$

measures, and likely represents the initial spike in impaired cerebrovascular reactivity after the primary insult, which then improves spontaneously over the next $48 \mathrm{~h}$ of ICU care. With that said, the TIL metrics provided are course measures of therapeutic intensity with documentation daily in our data set. It is unknown if more frequent intervention measures,

b

Mean Daily ICP vs. Daily TTIL

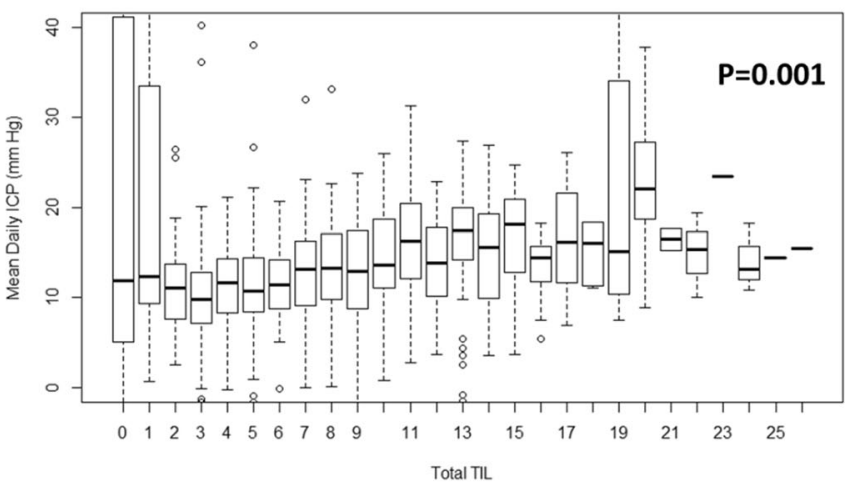

d

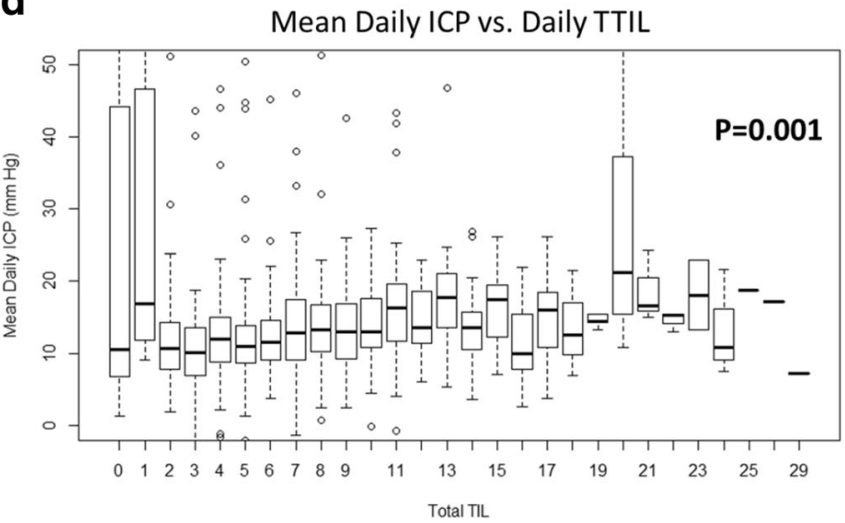

between $\%$ time and $\mathrm{PRx}>0$ was non-significant for both increasing and decreasing mean values. Plot of mean daily $\%$ time with PRx above 0 versus daily TTIL a; Plot of mean daily ICP versus daily TTIL $\mathbf{b}$; Plot of mean daily \% time with PRx above 0 versus daily TTIL for time-shifted data c; Plot of mean daily ICP versus daily TTIL for time-shifted data d 
Table 1 Mean daily $\%$ time with PRx $>0$, the Mann $U$ testing for significant TIL sub-scores

\begin{tabular}{|c|c|c|c|c|c|c|}
\hline \multirow[t]{3}{*}{ TIL sub-score } & \multicolumn{2}{|c|}{ Day-matched data } & \multirow[t]{3}{*}{ Mann $U p$ value } & \multicolumn{2}{|l|}{ Time-shifted data } & \multirow[t]{3}{*}{ Mann $U p$ value } \\
\hline & \multicolumn{2}{|c|}{ Mean $( \pm$ SD) daily $\%$ time with $\mathrm{PRx}>0$} & & \multicolumn{2}{|c|}{ Mean $( \pm$ SD) daily $\%$ time with $\mathrm{PRx}>0$} & \\
\hline & No intervention & Intervention & & No intervention & Intervention & \\
\hline Fluid (vasopressors) & $57.7(23.0)$ & $49.5(24.0)$ & $2.1 \times 10^{-8}$ & $57.0(23.2)$ & $47.8(23.4)$ & $1.7 \times 20^{-8}$ \\
\hline Hyperventilation (mild) & $55.7(24.6)$ & $48.0(22.6)$ & $2.5 \times 10^{-8}$ & $54.5(24.6)$ & $46.3(22.1)$ & $5.8 \times 10^{-8}$ \\
\hline Hypothermia (mild) & $52.8(24.0)$ & $44.5(22.4)$ & 0.0001 & $51.1(23.9)$ & $43.5(21.0)$ & 0.001 \\
\hline Sedation (High) & $54.2(23.4)$ & $49.0(24.4)$ & 0.0001 & $53.4(23.5)$ & $46.7(23.5)$ & $1.1 \times 10^{-6}$ \\
\hline
\end{tabular}

$C P P$, cerebral perfusion pressure; $I C P$, intra-cranial pressure; $M A P$, mean arterial pressure; $P R x$, pressure reactivity index (correlation between slowwaves in ICP and MAP); $S D$, standard deviation; TIL, therapeutic intensity level. This table reports $p$ values from the Mann $U$ testing, comparing mean daily \% time above PRx of 0 for specific TIL sub-scores. This table reports both the day-matched data sheet and the time-shifted data sheet, evaluating the difference in mean values between those receiving a specific intervention vs. those who did not. *Note: $p$ values are bolded for statistical significance after the Bonferroni correction. TIL fluid (vasopressor) refers to the need for vasopressor therapy to maintain CPP goals. TIL hyperventilation (mild) refers to mild hypocapnia for ICP control $\left(\mathrm{PaCO}_{2}=35\right.$ to $40 \mathrm{mmHg}$ ). TIL hypothermia (mild) refers to cooling to no lower than $35^{\circ} \mathrm{C}$. TIL sedation (high) refers to high sedation levels aimed at ICP control, but not burst suppression

perhaps accurately marked in the high-frequency physiology data, would provide stronger associations with changes in $\mathrm{PRx}$ metrics over time. Recent literature supports variance in mean PRx response after TBI, with those displaying positive global outcomes having a more rapid decline in PRx during the initial ICU days of care [22]. Thus, future analysis of the impact of therapeutic measures on cerebrovascular reactivity will not only benefit from high-resolution treatment data, but also various sub-group analyses based on age, sex, co-morbidities, injury pattern/burden, and patient global outcome. Such analyses will require large multi-center data sets to accomplish these goals.

Second, therapeutic intensity as measured through the total TIL score has little relationship with cerebrovascular reactivity, either in an analysis of contemporaneous data, or when lag effects are explored in time-shifted TIL data. This was also confirmed using the first 7 days and first 3 days of data, displaying identical results, indicating no difference between the entire ICU stay versus the initial acute $72 \mathrm{~h}$. These results were exemplified by the lack of statistically significant generalized and LME modeling between TTIL and all cerebrovascular reactivity metrics. This is an important finding, which suggests that overall, current ICU-based treatment strategies for TBI essentially have limited impact on cerebrovascular reactivity. This corroborates the findings of Donnelly et al. in a large single center retrospective cohort of over 1000 TBI patients, where despite changes in BTF-based ICP and CPP targets, little change in PRx or outcome was seen over 25 years [7].

Third, daily TIL sub-scores for most individual interventions failed to show any significant relationship with the PRx metrics we explored. Only four daily TIL sub-score parameters were found to be associated with lower mean daily PRx and daily $\%$ time with PRx above 0 . These included mild hyperventilation, mild hypothermia, high levels of sedation for ICP therapy, and vasopressor usage to target CPP. These results were similar for both the day-matched and time-shifted data sheets. Of note, the absolute reduction in $\%$ time with PRx above 0 was only modest for these 4 treatments, with less than $10 \%$ reduction. This highlights that the majority of currently applied therapies for TBI have limited impact on cerebrovascular reactivity. With that said, it must be acknowledged that given the strong link between impaired cerebrovascular reactivity and global patient outcome documented in various patient series $[5,7,20,28,29]$, even small changes in vascular reactivity metrics related to current TBI therapies may carry important implications for mortality and morbidity. It is still unclear how much of an improvement in PRx, or other cerebrovascular reactivity metrics, is required in order to leave a demonstrable impact of patient global outcome. Thus, despite the majority of TIL-measured therapies failing to demonstrate a significant change in PRx in this study, those interventions which had an impact may carry an important role moving forward in the initial modulation of cerebrovascular reactivity. Further to this, patient sub-group analysis based on injury pattern, injury burden, and age may play a role in individual patient response to current TBI therapies. As such, one should not walk away from this current work thinking "all is lost" regarding the impact of current TBI treatments on cerebrovascular reactivity. Much further work in this area is required.

Fourth, mild hyperventilation appeared to be associated with reduced mean daily PRx and daily \% time with PRx above 0 . This is in keeping with the literature body supporting improved vascular reactivity with mild hyperventilation $[15$, $16,18]$. However, more intensive daily hyperventilation, as assessed via other daily TIL sub-score measures (i.e., $\mathrm{PaCO}_{2}$ $<35 \mathrm{mmHg}$ ), failed to display any relationship with cerebrovascular reactivity [16]. This requires further investigation using treatment data with higher temporal resolution. 
However, the lack of association with cerebrovascular reactivity in the setting of more intensive hyperventilation may be secondary to exhaustion of the cerebrovascular reserve with such intensive reduction in $\mathrm{CO}_{2}$ levels [16].

Fifth, mild hypothermia (i.e., core temp $35-36.5^{\circ} \mathrm{C}$ ) was associated with a modest reduction in mean daily PRx and daily percentage of time above PRx of 0 , with more intensive hypothermia therapy (i.e., core temp below $35^{\circ} \mathrm{C}$ ) not being associated with vascular reactivity in this cohort. Stable/ improved cerebral autoregulatory capacity is known to occur with hypothermia therapy $[2,10,11]$. However, experimental models $[8,9,23]$, indicate that moderate to severe degrees of hypothermia can lead to loss of cerebrovascular reactivity. This may account for the lack of association between PRx variables and more intensive measures of hypothermia measures through other TIL sub-score metrics. This intimate relation between core and brain temperature requires further investigation using continuously measured cerebrovascular reactivity.

Sixth, high levels of sedation for ICP control were associated with a modest decrease in daily mean PRx and daily \% time above PRx of 0 . This likely represents a treatment effect on ICP, and subsequently CPP, with CPP values being obtained closer to the optimal CPP value $[1,17,21]$. However, sedation aimed at burst suppression, as assessed via a separate TIL sub-score value, was not associated with a change in PRx metrics. This may reflect injury/disease severity, or reflect the impairment in cerebrovascular reactivity seen with burst suppression in other patient cohorts using various anesthetic agents $[19,25]$. Further analysis of this relationship between sedative levels and cerebrovascular reactivity is required, using higher resolution data.

Seventh, the use of vasopressors to target CPP was also associated with a modest decrease in mean daily PRx and daily percentage of time with PRx above 0 . This likely reflects improved CPP targeting with vasopressors and avoiding CPP values below optimal CPP metrics. However, the impact of vasoactive compounds on cerebrovascular reactivity after TBI is still unclear, with many leading to vasoconstrictive states in experimental models [14], and requires future investigation using high temporal resolution vasopressor dosing data linked with high-frequency physiology in time-series.

Finally, the overall daily time spent with PRx above 0 was $40 \%$ or higher for all days, during the first 7 days after injury. This highlights the unchanged burden of impaired cerebrovascular reactivity after TBI, despite ongoing active ICP and CPP treatment. It further raises the questions of the role for individualized physiologic targets in TBI care, such as those posed by individual CPP optimum therapy [1, 17, 21], which is currently under prospective evaluation. Overall, this motivates further investigation into the molecular mechanisms involved in impaired cerebrovascular reactivity after TBI. Such analysis will require the integration of protein biomarkers with genetic profiling [30], in order to determine potential mechanisms driving impaired cerebrovascular reactivity. It is through such techniques that therapies directed at prevention and treatment may be developed, potentially leading to improved morbidity and mortality in TBI.

\section{Limitations}

The overall patient numbers with outcome and basic demographics were low at 249. This high-resolution cohort was a small specialty sub-cohort within the larger CENTER-TBI data collection scheme. This particular cohort of patients is unique in providing both high-frequency physiologic data and daily TIL scores during the acute phase of ICU stay, starting from the day of injury. Similar data will be needed to replicate our results, and more detailed assessments of the impact of interventions on autoregulatory efficiency will require therapy data with higher temporal resolution of treatment characteristics, such as TIL/TIL sub-scores, vasopressor dosing, and sedation dosing.

Furthermore, the impact of mild hyperventilation, mild hypothermia, high sedation levels, and vasopressor usage is still unclear. The impact on PRx metrics was modest at best in this analysis. Future studies require higher frequency data collection for TIL, core temperature, brain temperature, sedation dosing, and vasopressor dosing, in order to determine if any temporal response is seen between these treatments and cerebrovascular reactivity.

It must be acknowledged that TIL scores, and sub-scores, are relatively gross measures of therapeutic intensity. As such, despite failing to demonstrate a strong statistical significance with daily vascular reactivity metrics, therapeutic intensity measures captured at a higher temporal frequency, with more detailed annotations linked with the real-time physiology, may demonstrate an association with vascular reactivity measures. As such, the results of this study should be considered preliminary. Further studies are planned to evaluate the association between vascular reactivity, ICP, CPP, and other cerebral physiologic measures, using time-series physiology data with detailed treatment annotations.

Finally, in such a small data set, with various TIL-based treatment metrics measured, sometimes with very few patients have specific interventions, it raises the question of potential false-positive or false-negative results. We attempted to account for such false statistical positives through alpha correction using the Bonferonni methodology, only report results as significant with a $p$ value of less than 0.0005 . As such, the positive results reported, we feel are likely true positives. However, the significant reported TIL sub-scores were amongst the main drivers of overall TIL in our cohort (as seen in Appendix C). Thus, the overall significant association with PRx metrics may just be a reflection of these aspects being reported more frequently. In parallel, with such a small 
data set and large number of treatment variables, with a strict corrected alpha of 0.0005 , there exists the potential that some of the negative results may be false-negatives. As such, we emphasize the preliminary nature of our results here, and highlight the need for larger future studies with higher frequency treatment data. The results within this manuscript should be considered exploratory only at this time.

Given all the highlighted limitations, the results of this study should be considered preliminary, despite corroborating the previous retrospective work in the literature [7].

\section{Conclusions}

Cerebrovascular reactivity remains relatively independent of therapeutic intensity, suggesting inadequacy of current TBI therapies in modulating impaired autoregulation. These findings provide support for prior preliminary retrospective findings, and support the need for investigation into the molecular mechanisms involved in order to develop therapies aimed at prevention and treatment.

Acknowledgments CENTER-TBI high resolution sub-study participants and investigators: Audny Anke, Department of Physical Medicine and Rehabilitation, University hospital Northern Norway; Ronny Beer, Department of Neurology, Neurological Intensive Care Unit, Medical University of Innsbruck, Innsbruck, Austria; Bo-Michael Bellander, Department of Neurosurgery \& Anesthesia \& intensive care medicine, Karolinska University Hospital, Stockholm, Sweden; Andras Buki, Department of Neurosurgery, University of Pecs and MTA-PTE Clinical Neuroscience MR Research Group and Janos Szentagothai Research Centre, University of Pecs, Hungarian Brain Research Program, Pecs, Hungary; Giorgio Chevallard, NeuroIntensive Care, Niguarda Hospital, Milan, Italy; Arturo Chieregato, NeuroIntensive Care, Niguarda Hospital, Milan, Italy; Giuseppe Citerio, NeuroIntensive Care Unit, Department of Anesthesia \& Intensive Care, ASST di Monza, Monza, Italy; and School of Medicine and Surgery, Università Milano Bicocca, Milano, Italy; Endre Czeiter, Department of Neurosurgery, University of Pecs and MTA-PTE Clinical Neuroscience MR Research Group and Janos Szentagothai Research Centre, University of Pecs, Hungarian Brain Research Program (Grant No. KTIA 13 NAP-A-II/8), Pecs, Hungary; Bart Depreitere, Department of Neurosurgery, University Hospitals Leuven, Leuven, Belgium; George Eapen †, Shirin Frisvold, Department of Anesthesiology and Intensive Care, University Hospital Northern Norway, Tromso, Norway; Raimund Helbok, Department of Neurology, Neurological Intensive Care Unit, Medical University of Innsbruck, Innsbruck, Austria; Stefan Jankowski, Neurointensive Care, Sheffield Teaching Hospitals NHS Foundation Trust, Sheffield, UK; Daniel Kondziella, Departments of Neurology, Clinical Neurophysiology and Neuroanesthesiology, Region Hovedstaden Rigshospitalet, Copenhagen, Denmark; Lars-Owe Koskinen, Department of Clinical Neuroscience, Neurosurgery, Umea University Hospital, Umea, Sweden; Geert Meyfroidt, Intensive Care Medicine, University Hospitals Leuven, Leuven, Belgium; Kirsten Moeller, Department Neuroanesthesiology, Region Hovedstaden Rigshospitalet, Copenhagen, Denmark; David Nelson, Department of Neurosurgery \& Anesthesia \& intensive care medicine, Karolinska University Hospital, Stockholm, Sweden; Anna Piippo-Karjalainen, Helsinki University Central Hospital, Helsinki, Finland; Andreea Radoi, Department of Neurosurgery, Vall d'Hebron University Hospital, Barcelona, Spain;
Arminas Ragauskas, Department of Neurosurgery, Kaunas University of technology and Vilnius University, Vilnius, Lithuania; Rahul Raj, Helsinki University Central Hospital, Helsinki, Finland; Jonathan Rhodes, Department of Anaesthesia, Critical Care \& Pain Medicine NHS Lothian \& University of Edinburg, Edinburgh, UK; Saulius Rocka, Department of Neurosurgery, Kaunas University of technology and Vilnius University, Vilnius, Lithuania; Rolf Rossaint, Department of Anaesthesiology, University Hospital of Aachen, Aachen, Germany; Juan Sahuquillo, Department of Neurosurgery, Vall d'Hebron University Hospital, Barcelona, Spain; Oliver Sakowitz, Klinik für Neurochirurgie, Klinikum Ludwigsburg, Ludwigsburg, Germany; Department of Neurosurgery, University Hospital Heidelberg, Heidelberg, Germany; Ana Stevanovic, Department of Anaesthesiology, University Hospital of Aachen, Aachen, Germany; Nina Sundström, Department of Radiation Sciences, Biomedical Engineering, Umea University Hospital, Umea, Sweden; Riikka Takala, Perioperative Services, Intensive Care Medicine, and Pain Management, Turku University Central Hospital and University of Turku, Turku, Finland; Tomas Tamosuitis, Neuro-intensive Care Unit, Kaunas University of Health Sciences, Kaunas, Lithuania; Olli Tenovuo, Rehabilitation and Brain Trauma, Turku University Central Hospital and University of Turku, Turku, Finland; Peter Vajkoczy, Neurologie, Neurochirurgie und Psychiatrie, Charité-Universitätsmedizin Berlin, Berlin, Germany; Alessia Vargiolu, NeuroIntensive Care Unit, Department of Anesthesia \& Intensive Care, ASST di Monza, Monza, Italy; Rimantas Vilcinis, Department of Neurosurgery, Kaunas University of Health Sciences, Kaunas, Lithuania; Stefan Wolf, Interdisciplinary Neuro Intensive Care Unit, Charité-Universitätsmedizin Berlin, Berlin, Germany; Alexander Younsi, Department of Neurosurgery, University Hospital Heidelberg, Heidelberg, Germany.

Funding Data used in preparation of this manuscript were obtained in the context of CENTER-TBI, a large collaborative project with the support of the European Union 7th Framework program (EC grant 602150). Additional funding was obtained from the Hannelore Kohl Stiftung (Germany), from OneMind (USA) and from Integra LifeSciences Corporation (USA).

DKM was also supported by funding from the National Institute for Health Research (NIHR, UK) through a Senior Investigator award and the Cambridge Biomedical Research Centre at the Cambridge University Hospitals NHS Foundation Trust. The study also received additional support from the NIHR Clinical Research network. The views expressed are those of the authors and not necessarily those of the NHS, the NIHR or the Department of Health and Social Care, UK.

FAZ has received salary support for dedicated research time, during which this project was completed. FAZ receives research support from the University of Manitoba Thorlakson Chair in Surgical Research Establishment Fund, the University of Manitoba VPRI Research Investment Fund (RIF), the University of Manitoba Rudy Falk Clinician-Scientist Professorship, and the Health Sciences Centre Foundation Winnipeg.

\section{Compliance with ethical standards}

Conflict of interest Data used in preparation of this manuscript were obtained in the context of CENTER-TBI, a large collaborative project with the support of the European Union 7th Framework program (EC grant 602150). Additional funding was obtained from the Hannelore Kohl Stiftung (Germany), from OneMind (USA), and from Integra LifeSciences Corporation (USA).

PS and MC receive part of licensing fees for the software ICM+ (Cambridge Enterprise Ltd., UK) used for data collection and analysis in this study. 
Ethical approval All procedures performed in studies involving human participants were in accordance with the ethical standards of the institutional and/or national research committee (name of institute/committee) and with the 1964 Helsinki declaration and its later amendments or comparable ethical standards.

Data used in these analyses were collected as part of the CENTERTBI study which had individual national or local regulatory approval; the UK Ethics approval is provided as an exemplar: (IRAS No. 150943; REC 14/SC/1370). The CENTER-TBI study (EC grant 602150) has been conducted in accordance with all relevant laws of the EU if directly applicable or of direct effect and all relevant laws of the country where the recruiting sites were located, including but not limited to, the relevant privacy and data protection laws and regulations (the "Privacy Law"), the relevant laws and regulations on the use of human materials, and all relevant guidance relating to clinical studies from time to time in force including, but not limited to, the ICH Harmonised Tripartite Guideline for Good Clinical Practice (CPMP/ICH/135/95) ("ICH GCP") and the World Medical Association Declaration of Helsinki entitled "Ethical Principles for Medical Research Involving Human Subjects." Informed consent by the patients and/or the legal representative/next of kin was obtained, accordingly to the local legislations, for all patients recruited in the core dataset of CENTER-TBI and documented in the e-CRF.

Informed consent Informed consent was obtained from all individual participants included in the study. Informed consent by the patients and/or the legal representative/next of kin was obtained, accordingly to the local legislations, for all patients recruited in the core dataset of CENTER-TBI and documented in the e-CRF.

Open Access This article is distributed under the terms of the Creative Commons Attribution 4.0 International License (http:// creativecommons.org/licenses/by/4.0/), which permits unrestricted use, distribution, and reproduction in any medium, provided you give appropriate credit to the original author(s) and the source, provide a link to the Creative Commons license, and indicate if changes were made.

\section{References}

1. Aries MJH, Czosnyka M, Budohoski KP et al (2012) Continuous determination of optimal cerebral perfusion pressure in traumatic brain injury. Crit Care Med 40(8):2456-2463

2. Bisschops LLA, Hoedemaekers CWE, Simons KS, van der Hoeven JG (2010) Preserved metabolic coupling and cerebrovascular reactivity during mild hypothermia after cardiac arrest. Crit Care Med 38(7):1542-1547

3. Brady KM, Lee JK, Kibler KK, Easley RB, Koehler RC, Shaffner DH (2008) Continuous measurement of autoregulation by spontaneous fluctuations in cerebral perfusion pressure: comparison of 3 methods. Stroke 39(9):2531-2537

4. Carney N, Totten AM, O'Reilly C et al (2017) Guidelines for the management of severe traumatic brain injury, Fourth Edition. Neurosurgery 80(1):6-15

5. Czosnyka M, Smielewski P, Kirkpatrick P, Laing RJ, Menon D, Pickard JD (1997) Continuous assessment of the cerebral vasomotor reactivity in head injury. Neurosurgery 41(1):11-17 discussion 17-19

6. Doiron D, Marcon Y, Fortier I, Burton P, Ferretti V (2017) Software application profile: Opal and Mica: open-source software solutions for epidemiological data management, harmonization and dissemination. Int J Epidemiol 46(5):1372-1378

7. Donnelly J, Czosnyka M, Adams $\mathrm{H}$ et al (2018) Twenty-five years of intracranial pressure monitoring after severe traumatic brain injury: a retrospective, single-center analysis. Neurosurgery. https://doi.org/10.1093/neuros/nyy468

8. Fujita M, Wei EP, Povlishock JT (2012) Effects of hypothermia on cerebral autoregulatory vascular responses in two rodent models of traumatic brain injury. J Neurotrauma. 2019 May 15;36(10):15051517. https://doi.org/10.1089/neu.2018.6182

9. Irikura K, Miyasaka Y, Nagai S, Yuzawa I, Morii S, Fujii K (1998) Moderate hypothermia reduces hypotensive, but not hypercapnic vasodilation of pial arterioles in rats. J Cereb Blood Flow Metab 18(12):1294-1297

10. Koizumi H, Suehiro E, Fujiyama Y, Yoneda H, Ishihara H, Nomura S, Fujii M, Suzuki M (2016) Effects of brain temperature on cerebrovascular autoregulation during the acute stage of severe traumatic brain injury. Acta Neurochir Suppl 122:193-197

11. Lavinio A, Timofeev I, Nortje J et al (2007) Cerebrovascular reactivity during hypothermia and rewarming. Br J Anaesth 99(2):237244

12. Maas AIR, Harrison-Felix CL, Menon D et al (2011) Standardizing data collection in traumatic brain injury. J Neurotrauma 28(2):177187

13. Maas AIR, Menon DK, Steyerberg EW, Citerio G, Lecky F, Manley GT, Hill S, Legrand V, Sorgner A, CENTER-TBI Participants and Investigators (2015) Collaborative European NeuroTrauma Effectiveness Research in Traumatic Brain Injury (CENTERTBI): a prospective longitudinal observational study. Neurosurgery 76(1):67-80

14. McCulloch J, Edvinsson L (1984) Cerebrovascular smooth muscle reactivity: a critical appraisal of in vitro and in situ techniques. J Cereb Blood Flow Metab 4(2):129-139

15. McCulloch TJ, Turner MJ (2009) The effects of hypocapnia and the cerebral autoregulatory response on cerebrovascular resistance and apparent zero flow pressure during isoflurane anesthesia. Anesth Analg 108(4):1284-1290

16. Meng L, Gelb AW (2015) Regulation of cerebral autoregulation by carbon dioxide. Anesthesiology 122(1):196-205

17. Needham E, McFadyen C, Newcombe V, Synnot AJ, Czosnyka M, Menon D (2017) Cerebral perfusion pressure targets individualized to pressure-reactivity index in moderate to severe traumatic brain injury: a systematic review. J Neurotrauma 34(5):963-970

18. Newell DW, Weber JP, Watson R, Aaslid R, Winn HR (1996) Effect of transient moderate hyperventilation on dynamic cerebral autoregulation after severe head injury. Neurosurgery 39(1):35-43 discussion 43-44

19. Reinsfelt B, Westerlind A, Houltz E, Ederberg S, Elam M, Ricksten S-E (2003) The effects of isoflurane-induced electroencephalographic burst suppression on cerebral blood flow velocity and cerebral oxygen extraction during cardiopulmonary bypass. Anesth Analg 97(5):1246-1250

20. Sorrentino E, Diedler J, Kasprowicz M et al (2012) Critical thresholds for cerebrovascular reactivity after traumatic brain injury. Neurocrit Care 16(2):258-266

21. Steiner LA, Czosnyka M, Piechnik SK, Smielewski P, Chatfield D, Menon DK, Pickard JD (2002) Continuous monitoring of cerebrovascular pressure reactivity allows determination of optimal cerebral perfusion pressure in patients with traumatic brain injury. Crit Care Med 30(4):733-738

22. Svedung Wettervik T, Howells T, Enblad P, Lewén A (2019) Temporal neurophysiological dynamics in traumatic brain injury: role of pressure reactivity and optimal cerebral perfusion pressure for predicting outcome. J Neurotrauma. https://doi.org/10.1089/ neu.2018.6157

23. Verhaegen MJ, Todd MM, Hindman BJ, Warner DS (1993) Cerebral autoregulation during moderate hypothermia in rats. Stroke 24(3):407-414

24. Weersink CSA, Aries MJH, Dias C et al (2015) Clinical and physiological events that contribute to the success rate of finding 
"optimal" cerebral perfusion pressure in severe brain trauma patients. Crit Care Med 43(9):1952-1963

25. Woodcock TE, Murkin JM, Farrar JK, Tweed WA, Guiraudon GM, McKenzie FN (1987) Pharmacologic EEG suppression during cardiopulmonary bypass: cerebral hemodynamic and metabolic effects of thiopental or isoflurane during hypothermia and normothermia. Anesthesiology 67(2):218-224

26. Zeiler FA, Donnelly J, Calviello L, Smielewski P, Menon DK, Czosnyka M (2017) Pressure autoregulation measurement techniques in adult traumatic brain injury, part II: a scoping review of continuous methods. J Neurotrauma 34(23):3224-3237

27. Zeiler FA, Donnelly J, Smieleweski P, Menon D, Hutchinson PJ, Czosnyka M (2018) Critical thresholds of ICP derived continuous cerebrovascular reactivity indices for outcome prediction in noncraniectomized TBI patients: PRx, PAx and RAC. J Neurotrauma 35(10):1107-1115

28. Zeiler FA, Ercole A, Cabeleira M, Carbonara M, Stocchetti N, Menon DK, Smielewski P, Czosnyka M (2018) Comparison of performance of different optimal cerebral perfusion pressure parameters for outcome prediction in adult TBI: a CENTER-TBI study. $\mathrm{J}$ Neurotrauma

29. Zeiler FA, Ercole A, Cabeleira M, Zoerle T, Stocchetti NN, Menon DK, Smielewski P, Czosnyka M, CENTER-TBI High Resolution Sub-Study Participants and Investigators (2019) Univariate comparison of performance of different cerebrovascular reactivity indices for outcome association in adult TBI: a CENTER-TBI study. Acta Neurochir. https://doi.org/10.1007/s00701-019-038441

30. Zeiler FA, Thelin EP, Donnelly J, Stevens AR, Smielewski P, Czosnyka M, Hutchinson PJ, Menon DK (2019) Genetic drivers of cerebral blood flow dysfunction in TBI: a speculative synthesis. Nat Rev Neurol 15(1):25-39

31. Zuercher P, Groen JL, Aries MJH, Steyerberg EW, Maas AIR, Ercole A, Menon DK (2016) Reliability and validity of the therapy intensity level scale: analysis of clinimetric properties of a novel approach to assess management of intracranial pressure in traumatic brain injury. J Neurotrauma 33(19):1768-1774

George Eapen is deceased. This paper is dedicated to his memory.

Publisher's note Springer Nature remains neutral with regard to jurisdictional claims in published maps and institutional affiliations.

\section{Affiliations}

\section{Frederick A. Zeiler ${ }^{1,2,3,8,9}$ (D) Ari Ercole $^{1} \cdot$ Erta Beqiri $^{3} \cdot$ Manuel Cabeleira $^{3} \cdot$ Marcel Aries $^{4} \cdot$ Tommaso Zoerle $^{5}$. Marco Carbonara ${ }^{5} \cdot$ Nino Stocchetti ${ }^{5,6} \cdot$ Peter Smielewski $^{3} \cdot$ Marek Czosnyka $^{3,7} \cdot$ David K. Menon $^{1}$}

1 Division of Anaesthesia, Addenbrooke's Hospital, University of Cambridge, Cambridge, UK

2 Department of Surgery, Rady Faculty of Health Sciences, University of Manitoba, Winnipeg, MB R3A 1R9, Canada

3 Brain Physics Laboratory, Division of Neurosurgery, Department of Clinical Neurosciences, Addenbrooke's Hospital, University of Cambridge, Cambridge, UK

4 Department of Intensive Care, Maastricht UMC, Maastricht, Netherlands

5 Neuro ICU Fondazione IRCCS Cà Granda Ospedale Maggiore Policlinico, Milan, Italy
6 Department of Physiopathology and Transplantation, Milan University, Milan, Italy

7 Institute of Electronic Systems, Warsaw University of Technology, Warsaw, Poland

8 Department of Human Anatomy and Cell Science, Rady Faculty of Health Sciences, University of Manitoba, Winnipeg, Canada

9 Department of Biomedical Engineering, Faculty of Engineering, University of Manitoba, Winnipeg, Canada 